MEMORY RESPONSES

\title{
Fitter, faster, better innate immune cells
}

Innate immune cells are essential for sensing infection and initiating immune responses against microbial pathogens. Now, Soudja et al. report that successful memory responses in vaccinated hosts depend on interferon- $\gamma$ (IFN $\gamma)$ that is secreted by memory $\mathrm{T}$ cells, which directs the recruitment, activation and effector function of innate myeloid and lymphoid cells.

The authors immunized wild-type mice with either phosphate buffered saline or Listeria monocytogenes and then challenged them with L. monocytogenes 5-6 weeks later. In vaccinated mice, $\mathrm{LY}_{6 \mathrm{C}}{ }^{+}$monocytes had already differentiated into effector cells 8 hours after challenge infection. Faster activation of neutrophils,

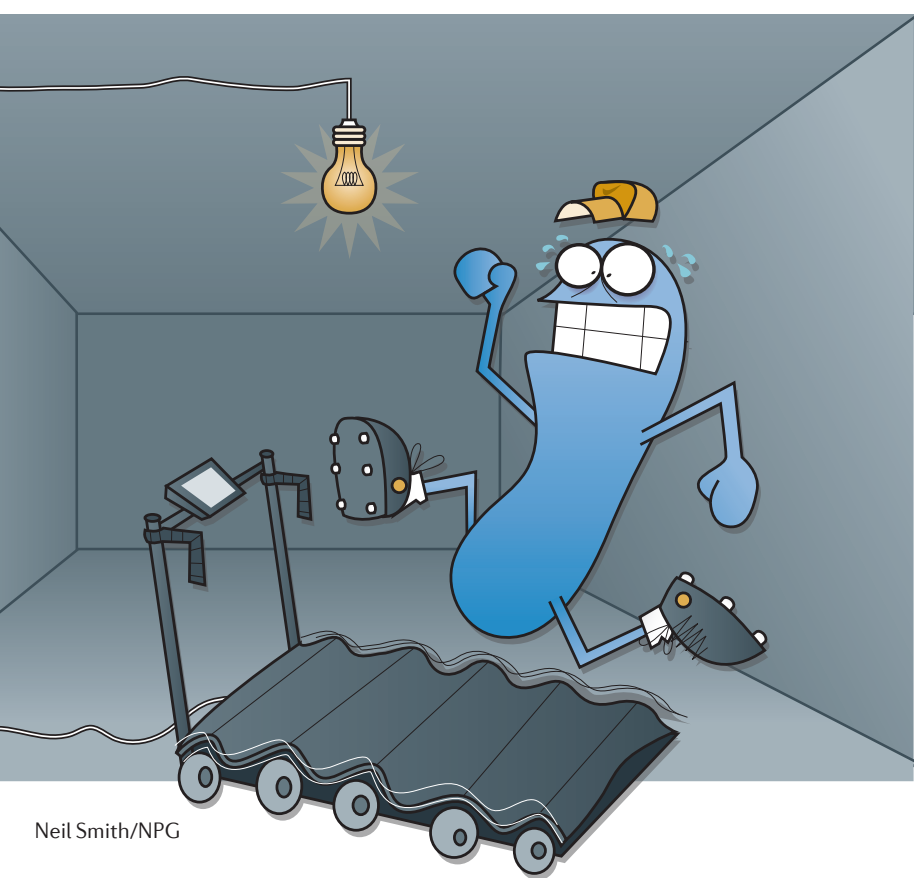

tissue macrophages, dendritic cells (DCs), natural killer (NK) cells and NKT cells was also observed in vaccinated mice compared with naive mice.

Next, the authors analysed spleen tissue sections and showed that the activation of innate cells was associated with enhanced recruitment of these cells from the blood to the infected spleen in vaccinated mice. Clusters of cells (including CD11 b ${ }^{+}$ cells and CC-chemokine receptor 2 (CCR2)-expressing monocytes, as well as memory T cells and NK cells) were detected in the red pulp in the spleens of vaccinated mice 8 hours after infection but this was delayed in naive mice.

To determine whether these differences in naive versus vaccinated mice also altered the activation of pathogen-specific naive T cells, the authors transferred ovalbumin (OVA)-specific CD8 ${ }^{+} \mathrm{T}$ cells (OT-I cells) into naive or vaccinated mice. The mice were challenged with OVA-expressing attenuated L. monocytogenes. After 7 days, OT-I cells had differentiated into effector cells in naive mice, whereas OT-I cells that were transferred into L. monocytogenes-vaccinated mice developed a memory phenotype.

So, are memory T cells crucial for the changes that are observed in innate immune cells in vaccinated mice? The depletion of both $\mathrm{CD} 4^{+}$ and $\mathrm{CD}^{+} \mathrm{T}$ cells before challenge infection prevented the early activation of innate cells ( 8 hours after infection) in vaccinated mice.

To determine whether IFN $\gamma$ induced signalling in innate cells is important during memory responses, the authors generated chimeric mice lacking the IFN $\gamma$ receptor (IFN $\gamma \mathrm{R}$ ) or the type I IFN receptor (IFNAR1). When these mice were immunized and challenged with L. monocytogenes, the differentiation of both $\mathrm{LY}^{+} \mathrm{C}^{+}$monocytes and neutrophils that lacked the IFN $\gamma$ receptor - but not those that lacked the IFNAR1 - was impaired in the spleen and other infected organs 8 hours after challenge infection. Interestingly, the differentiation of NK cells in IFN $\gamma$ R-deficient mice was delayed but not prevented, which suggests that indirect IFN $\gamma$-dependent signals enhance NK cell activation. The recruitment of innate cells into infected tissues was not affected. IFN $\gamma$ signalling activated monocytes through an IFN-regulatory factor 1 (IRF1)-independent mechanism and synergized with microbial sensing pathways to potentiate innate cell recruitment and activation. Gene expression analysis of $\mathrm{LY} \mathrm{C}^{+}$ monocytes showed specific responses to IFN $\gamma$ in vaccinated mice (including the expression of guanylate-binding proteins that are implicated in defence against intracellular bacteria) compared with a more general inflammatory response in naive mice.

Finally, the selective depletion of specific cell types in vaccinated chimeric mice lacking the IFN $\gamma \mathrm{R}$ showed that monocytes, DCs and macrophages are important for protective recall responses to L. monocytogenes infection.

Taken together, these results reveal that IFN $\gamma$ produced by memory $\mathrm{T}$ cells coordinates innate immune responses during infection in vaccinated hosts.

Elaine Bell

ORIGINAL RESEARCH PAPER Soudja, S. M. et al Memory-T-cell-derived interferon- $\gamma$ instructs potent innate cell activation for protective immunity. Immunity 40, 974-988 (2014) 